\title{
BMJ Open Adverse childhood experiences (ACEs) and cardiovascular development from childhood to early adulthood: study protocol of the Niagara Longitudinal Heart Study
}

\author{
Terrance J Wade, ${ }^{\oplus 1,2,3}$ Deborah D O'Leary, ${ }^{1,2}$ Kylie S Dempster, ${ }^{1,2}$ \\ Adam J MacNeil, ${ }^{1}$ Danielle S Molnar, ${ }^{3}$ Jennifer McGrath, ${ }^{4}$ John Cairney ${ }^{5}$
}

To cite: Wade TJ, O'Leary DD, Dempster KS, et al. Adverse childhood experiences (ACEs) and cardiovascular development from childhood to early adulthood: study protocol of the Niagara Longitudinal Heart Study. BMJ Open 2019;9:e030339. doi:10.1136/ bmjopen-2019-030339

- Prepublication history for this paper is available online. To view these files, please visit the journal online (http://dx.doi. org/10.1136/bmjopen-2019030339).

Received 9 March 2019

Revised 28 May 2019

Accepted 21 June 2019

Check for updates

(C) Author(s) (or their employer(s)) 2019. Re-use permitted under CC BY-NC. No commercial re-use. See rights and permissions. Published by BMJ.

For numbered affiliations see end of article.

Correspondence to

Dr Terrance J Wade;

twade@brocku.ca

\section{ABSTRACT}

Introduction Recent reviews have found substantial links between a toxic childhood environment including child abuse and severe household dysfunction and adult cardiovascular disease (CVD). Collectively referred to as adverse childhood experiences (ACEs), this toxic environment is prevalent among children, with recent Canadian estimates of child abuse at $27 \%-32 \%$, and severe household dysfunction at $49 \%$. Based on these prevalence rates, the potential effect of ACEs on CVD is more significant than previously thought. Yet, how ACEs amplify the risk for later CVD remains unclear. Lifestyle risk factors only partially account for this connection, instead directing attention to the interaction between psychosocial factors and physiological mechanisms such as inflammation. The Niagara Longitudinal Heart Study (NLHS) examines how ACEs influence cardiovascular health (CVH) from childhood to early adulthood. Integrating the stress process and biological embedding models, this study examines how psychosocial and physiological factors in addition to lifestyle factors explain the relationship between ACEs and CVH.

Methods This follow-up study combines three baseline studies from 2007 to 2012 that collected CVH measures including child blood pressure, heart rate, left ventricular structure and function, arterial stiffness indices and baroreflex sensitivity on 564 children. Baseline data also include anthropometric, biological, lifestyle, behavioural, and psychosocial measures that varied across primary studies. Now over 18 years of age, we will recruit and retest as many participants from the baseline studies as possible collecting data on ACEs, CVH, anthropometric, lifestyle and psychosocial measures as well as blood, saliva and hair for physiological biostress markers

Ethics and dissemination Ethics approval has been received for the NLHS follow-up. Written consent to participate in the follow-up study is obtained from each participant. Results testing all proposed hypotheses will be submitted for publication in peer-reviewed journals.

\section{INTRODUCTION}

A toxic childhood environment, referred to as adverse childhood experiences (ACEs), includes physical, sexual and emotional

\section{Strengths and limitations of this study}

- The same standardised methods and measures to assess cardiovascular health are used across all three baseline studies ensuring comparability of data across studies and over time.

- Data are collected on lifestyle, psychosocial and physiological markers identified in previous research linking adverse childhood experiences and cardiovascular disease in adults.

- This study focuses on a non-clinical, population-based sample.

- Lifestyle, behavioural, psychosocial, mental health and biostress measures vary across baseline studies.

- While retrospective measures of childhood adversity are collected from all participants, prospective, parent-reported measures of childhood adversity are only available on a subset of participants and exclude child maltreatment.

abuse, severe household dysfunction including substance abuse or serious mental illness, forced separation from parents and family and severe neglect, exposure to violence and crime in the home or neighbourhood, material disadvantage or homelessness, natural disasters and serious illnesses, accidents or injuries. ${ }^{1-3}$ These exposures are linked to adult physical health issues and diseases as well as other outcomes including poor school performance, mental disorder, criminality, substance abuse and general life instability. ${ }^{1-3}$ Recent reviews have found links between ACEs and adult cardiovascular disease (CVD). ${ }^{4-11}$ It is largely thought that child abuse, neglect and severe household dysfunction are rare occurrences and their effect on the overall health of the population is minimal. However, child adversity is common, with recent retrospective Canadian 
estimates of child abuse at 27\%-32\%, ${ }^{12-14}$ and severe household dysfunction at $49 \% .{ }^{13}$ Since CVD is a leading cause of death, the potential effect of ACEs is far more significant than formerly thought based on these prevalence rates. ${ }^{12} 13$ Yet, how ACEs amplify the risk for CVD later in life remains unclear.

\section{Linking ACEs to CVD: retrospective ACEs studies on adult populations}

Most ACEs studies have been conducted on adult populations finding a moderate to strong link with adult blood pressure (BP) and CVD endpoints including myocardial infarction, stroke, and ischaemic and coronary heart disease. ${ }^{1}$ 2 4-12 15-18 However, these studies are limited in three ways. First, they focus on decades-long, retrospective recall of ACEs which may be under-reported. ${ }^{1} 12$ While work supports the reliability of long-term recall, ${ }^{19}$ others argue that it is essential to get ACEs information as early in the life course as possible for accuracy and reliability of recall. ${ }^{19-21}$ Second, these studies are dependent on whether a cardiovascular condition or CVD has been diagnosed. Many people with heart conditions or CVD may not yet have a diagnosis leading to a bias of under-reporting. Third, these studies provide a one-time snapshot that does not permit examination of the developmental process of cardiovascular health (CVH) leading to CVD. This cross-sectional design impedes their ability to examine development of the cardiovascular system long before CVD is identified limiting their ability to provide causal explanations of why ACEs are linked to CVD.

\section{Linking ACEs to CVD: long-term longitudinal studies of child cardiovascular development}

Long-term, longitudinal studies of cardiovascular development provide one opportunity to address the limitations outlined above. Several long-term prospective studies beginning in the $1970 \mathrm{~s}, 80 \mathrm{~s}$ and 90 s identify childhood origins of heart disease. ${ }^{15} 1618{ }^{22-24}$ While these studies provide knowledge about the precursors of CVD, they have two principal limitations. First, they began prior to research identifying ACEs as a risk factor and collected these data much later, suffering the same limitation of long-term recall identified in restrospective ACEs studies. However, they still report significant connections between ACEs and CVH. For example, the Georgia Stress and Heart Study, beginning in 1989, examined changes in BP in 16 waves over 20 years in a growth curve analysis but they only measured ACEs in wave 15 (2008-2010). ${ }^{16}$ They found a significant interaction between ACE score and faster rising systolic and diastolic BP (SBP and DBP) after age 30. Most importantly, they found the difference in BP was not explained by traditionally identified risk factors, specifically physical activity, body mass index, smoking and family history of disease. They argue that other pathways may mediate the effect of ACEs on CVH through dysregulation of BP, specifically, the hypothalamic-pituitary-adrenal axis (HPA), sympathetic-adrenal-medullary axis (SAM) and elevated levels of inflammation. ${ }^{16}$
Second, these studies are limited by cardiovascular measures collected in the early waves during childhood and young adulthood. Initially, these studies focused on BP which, as noted above, may not manifest differences until much later in life ${ }^{16}$ while the studies that did collect more sensitive markers of cardiovascular structure and function predictive of future CVD did so in much later waves when participants were adults. ${ }^{22-27}$ This limits their ability to examine developmental changes in measures of CVH prospectively from childhood to early adulthood when persons appear to be more predisposed biologically to the negative effects of ACEs. ${ }^{28}$

\section{Summary and synthesis}

While the above studies are informative, we are left with a significant mechanistic gap and an incomplete conceptual framework, hindering our understanding of how ACEs and CVD are linked. Moreover, much of this research does not account for other childhood risk factors such as family socioeconomic status and parental health behaviours nor the accumulation and/or interaction of ACEs with these other factors. ${ }^{29-34}$ Many of these additional childhood stressors such as poverty and bullying have been included in an expanded view of ACEs. ${ }^{1-3}$ Finally, most research attempting to explain the connection between ACEs and CVD are domain specific, examining the relationship through (1) behaviour/ lifestyle processes, ${ }^{29}$ (2) physiological systems ${ }^{30} 31$ or (3) positive and negative psychosocial and mental health factors. ${ }^{72} 303234$ In this study, we integrate these domains in a multidimensional, prospective analysis to identify intersections between these domains and cardiovascular development. The importance of incorporating this multidimensional focus is supported by recent ACEs research and reviews that show that previously identified traditional and behavioural risk factors including family history, smoking, physical activity, alcohol use and obesity, while important in their own right, do not explain away the relationship between ACEs and CVD. ${ }^{4-11}$

But what is unique about this connection between ACEs and CVH that traditional risk factors are unable to explain? Some reviews point to psychosocial and mental health factors such as depressed affect, anger and social stress as mediating mechanisms. ${ }^{4}$ These findings direct research to focus on psychosocial factors that mediate the connection between ACEs and CVD. The stress process model $^{35}{ }^{36}$ provides a framework outlining how social stressors affect health outcomes and the role of psychosocial risk and protective factors. ${ }^{37}$ Aligning this model with cardiovascular research, studies have found that both depressed affect and anger were stronger mediators than traditional lifestyle risk factors (ie, smoking, physical activity, obesity, diabetes and hypertension) explaining the association between ACEs and ischaemic heart disease. ${ }^{7}$

Other studies and reviews that focus on the physiological mechanisms linking ACEs and CVD also redirect us back to psychosocial factors by proposing a process 


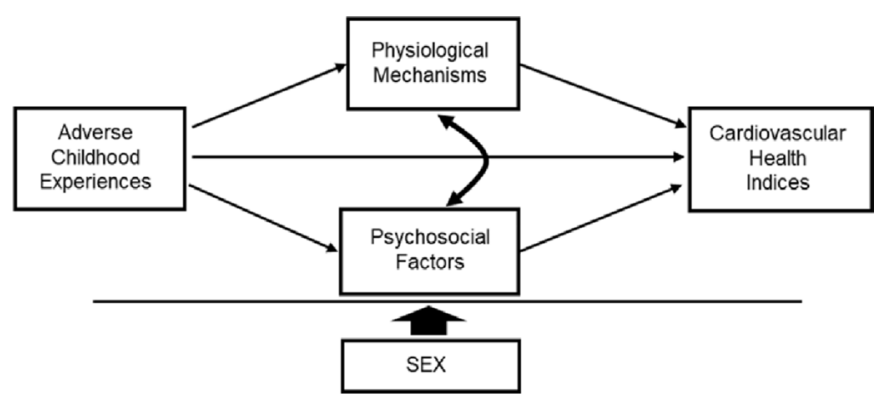

Figure 1 Conceptual linkages between ACEs and $\mathrm{CVH}$ through psychosocial and physiological factors. ACEs, adverse childhood experiences; $\mathrm{CVH}$, cardiovascular health.

of amplified inflammatory responses to social stress as adults. ${ }^{8-11}$ These studies suggest a biological embedding model $^{28}$ or psychoneuroimmunology model ${ }^{8}$ in which the embedding of stress into cells that regulate inflammation during ACEs in childhood through the HPA and/or SAM axis results in an elevated, chronic proinflammatory state altering the adaptive immune responses to future stressors affecting one's CVH trajectory. Consistent with this process, two reviews argue future research is necessary to link physiological and psychosocial domains. One review suggests a life course interaction between psychosocial and physiological processes through emotional problems linking ACEs to CVD. In essence, this creates a heightened, lifelong physiological susceptibility to social stress resulting in a chronic arousal of stress response systems. ${ }^{10}$ The other review examining ACEs in relation to biomarkers, specifically $\mathrm{C}$ reactive protein (CRP), interleukin-6 (IL-6) and fibrinogen measured in childhood, identified a mediational psychobiological pathway linking elevated acute inflammatory responses to psychosocial stress. ${ }^{9}$ This is further supported by evidence from animal and human studies that identify early stress as disrupting the HPA and SAM axes, ${ }^{38-41}$ and adaptive immune functions ${ }^{42} 43$ lending support to the biological embedding model. ${ }^{28}$ And while inflammation is normally tightly controlled by the body, when inflammation becomes chronic, pathological tissue damage results. For example, the Dunedin study showed a link between ACEs and CRP among adults at 32 years of age, a strong marker of CVH and a key mediator of inflammatory events. ${ }^{44}$ In another study, IL-6 was detected at elevated levels in adults with child maltreatment histories. ${ }^{45}$ These proinflammatory mediators accelerate harmful adaptations in cardiovascular structure and function, leading ultimately to heart disease/stroke. ${ }^{12846}$

\section{Study objectives and hypotheses to be tested}

Combining the stress process model ${ }^{34-37}$ and the biological embedding model, ${ }^{28}$ we investigate the psychosocial and physiological mediating pathways between ACEs and $\mathrm{CVH}$. For the stress process model, we focus on both positive and negative psychosocial factors. For the biological embedding model, we focus on adaptive stress responses and inflammation identified as important in the literature, ${ }^{1}$ 7-11 383947 specifically chronic cortisol levels, CRP, cytokines regulating inflammation such as IL-1 $\beta$, IL-2, IL-4, IL-6, IL-8 and IL-10, interferon- $\gamma$ (IFN- $\gamma$ ) and tumour necrosis factor (TNF $\alpha)$. We also examine premature cellular ageing as measured by telomere length which has been associated with ACEs, ${ }^{48}{ }^{49}$ inflammation, and $\mathrm{CVD}^{50}$ (see figure 1 ).

This study will prospectively test these pathways building on baseline data from three previous research projects (see methods section below). In our previous work examining baseline data in one of these studies, parent-reported ACEs predicted early obesity, increased waist circumference and elevated heart rate (HR), ${ }^{51}$ and systemic arterial stiffness but only among boys. ${ }^{52}$ Because these were parent-reported ACEs when the participants were 11-13 years, they did not include abuse but focused on parent-reported ACEs more generally. In this study, we collect self-reported ACEs from the participants who are now young adults.

To our knowledge, the effect of ACEs on preclinical CVH indices has not been studied in a prospective manner from childhood to early adulthood. The hypotheses of the study are:

1. Greater exposure to ACEs will result in lowered arterial compliance, distensibility and baroreflex sensitivity as well as elevated BP, HR, carotid artery intima-media thickness (IMT), left ventricular mass (LVM) and pulse-wave velocity going from childhood to early adulthood. ${ }^{4}$

2. The relationship between ACEs and changes in CVH will be mediated by psychosocial and/or physiological factors. ${ }^{4-9}$ Elevated physiological markers will mediate the relationship between changes in psychosocial factors and changes in $\mathrm{CVH}^{10}{ }^{114048}$ Those with elevated levels of inflammatory markers will be more susceptible to changes in psychosocial factors (eg, social stress) linked to CVH (ie, moderation-biological embedding hypothesis making one physiologically less able to adapt to environmental stress). ${ }^{10114048}$

3. The relationship between ACEs and CVH will differ across males and females. ${ }^{452}$ The process linking ACEs to changes in $\mathrm{CVH}$ through psychosocial and physiological factors will differ across males and females. ${ }^{452}$

\section{METHODS}

The Niagara Longitudinal Heart Study (NLHS) builds on three previously funded research projects in the Niagara region, Canada, from 2007 to 2012. Each baseline study varied in its collection of demographic, psychosocial, lifestyle and biological measures (see table 1). However, all three studies used the same standardised methods to assess CVH ensuring comparability of data across studies and over time (see table 2). The Health Behavioural and Environmental Assessment Team (HBEAT) Study also collected parent-reported ACEs. ${ }^{51} 52$

Study 1: The HBEAT study was conducted in two phases, from 2007 to 2012, The first phase was cross-sectional 
Table 1 Disposition and uniformity of measurement domains for NLHS follow-up study and cardiovascular baseline study subsamples across three Niagara area studies

\begin{tabular}{|c|c|c|c|c|}
\hline & \multirow{2}{*}{$\begin{array}{l}\text { Wave } 2 \\
\text { NLHS }\end{array}$} & \multicolumn{3}{|l|}{ Baseline (wave 1) } \\
\hline & & HBEAT & PHAST & BAM \\
\hline \multicolumn{5}{|l|}{ Sample disposition } \\
\hline Population & & Local school district & Local school district & Community \\
\hline Study design & & $\begin{array}{l}\text { Cross-sectional } \\
\text { (longitudinal) samples }\end{array}$ & Longitudinal sample & Longitudinal sample \\
\hline Cardiovascular laboratory subsample & $\begin{array}{l}\text { TBD } \\
\text { (Pilot } n=76 ; \text { Max } n=564 \text { ) }\end{array}$ & $334(110)$ & 126 & 104 \\
\hline Lab subsample age (years) & TBD & $10-14$ & $12-15$ & $8-18$ \\
\hline Lab subsample stratification & - & $\mathrm{BP}$ & rDCD & None \\
\hline \multicolumn{5}{|l|}{ Measurement domains } \\
\hline Family history of cardiovascular health & $\mathrm{X}(\mathrm{S})$ & $X(P)$ & $X(P)$ & - \\
\hline Lipid profile & $x$ & $x$ & $x$ & $\mathrm{X}$ \\
\hline $\begin{array}{l}\text { Physiological stress biomarkers (blood } \\
\text { serum) }\end{array}$ & $x$ & - & - & $\mathrm{X}$ \\
\hline Anthropometric measures & $\mathrm{X}$ & $x$ & $\mathrm{X}$ & $\mathrm{X}$ \\
\hline Lifestyle and behaviour measures & $X(S)$ & $X(S, P)$ & $X(S, P)$ & $X(S)$ \\
\hline Psychosocial measures & $X(S)$ & $X(S)$ & $X(S)$ & - \\
\hline Medical history & $X(S)$ & $X(P)$ & $X(P)$ & $X(S, P)$ \\
\hline
\end{tabular}

*Parent-reported ACEs exclude maltreatment items relating to sexual, physical and emotional abuse.

BAM, Brock Active Movement; BP, blood pressure; HBEAT, Heart Behavioural and Environmental Assessment Team; max N, maximum sample size (assuming 100\% recontact and recruitment); NLHS, Niagara Longitudinal Heart Study; P, parent-report data; PHAST, Physical Health and Activity Study Team; rDCD, at risk for developmental coordination disorder; S, self-report data; TBD, to be determined; X, data collected.

examining the social determinants of childhood BP. It included a large, school-based sample of 1913 children aged 11-14 years (grades 6-8) across 50 schools in a local school board. Of these, 1285 completed both a parent and child survey. From these, 224 were selected for detailed cardiovascular assessments stratified across BP levels of the 1913 children (high BP-BP $\geq 95$ th percentile; high-normal BP-BP $<95$ th percentile and $\geq 90$ th percentile; normal BP-BP $<90$ th percentile adjusting for age, sex and height). The second phase was an intervention study across 10 of the 50 schools ( 5 intervention and 5 comparison schools). In this phase, 647 students had their BP measured in spring, 2011 in grades 5-7 (10-13 years of age) of which 551 completed both parent and child questionnaires. Stratified using the above cut-points, 110 participants were selected equally across the intervention and control schools for detailed cardiovascular assessments. Students were then tested again in spring, 2012 (grades 6-8) and again completed the parent and child questionnaires. Combining both phases, the total HBEAT laboratory subsample for the NLHS includes a total possible 334 children. The battery of additional measures included family demographics, family history of CVD and related diseases, anthropometric measures, lifestyle, physical activity and a broad array of psychosocial and mental health measures. The 224 from phase 1 also had a 12 hours fasting lipid profile and the 110 from phase 2 are measured longitudinally having two annual waves of data during the transition from grades 5-7 to grades 6-8 (table 1).

Study 2: Physical Health and Activity Study Team (PHAST) was a 10-wave study conducted from 2004 to 2010 . The first wave screened a grade 4 cohort (8-9years) in fall, 2004 from 75 of 92 schools $(81.5 \%)$ in a local school board for developmental coordination disorder (DCD) to examine its connection to health and activity. Students were tracked and tested twice per year up to the 2006/2007 school year. In fall 2007, testing was done annually. In fall, 2007, a laboratory subsample of 126 participants were selected when they were 12-14 years (grades 7-8) stratified equally across those at risk for DCD $(\mathrm{rDCD}) \quad(\mathrm{n}=63)$ and a sex, school and grade-matched 
Table 2 Niagara Longitudinal Heart Study measures across key concepts*

\begin{tabular}{|c|c|c|c|}
\hline $\begin{array}{l}\text { Anthropometric and } \\
\text { cardiovascular }\end{array}$ & $\begin{array}{l}\text { Psychosocial resources and } \\
\text { mental health }\end{array}$ & Physiological stress biomarkers & Lifestyle and behavioural \\
\hline $\begin{array}{l}\text { Anthropometrics: } \\
\text { Height; body mass; waist } \\
\text { circumference; hip circumference; } \\
\text { fat mass; fat-free mass; body fat } \\
\text { percentage } \\
\text { Cardiovascular indices-Sitting: } \\
\text { Resting oscillometric SBP, DBP, } \\
\text { MAP, PP and HR. } \\
\text { Cardiovascular indices-Supine: } \\
\text { Resting manual SBP, DBP, } \\
\text { MAP, PP; beat-by-beat BP, HR } \\
\text { and R-R interval; cvBRS; CCA } \\
\text { IMT; arterial distensibility and } \\
\text { compliance; central pulse wave } \\
\text { velocity (PWV) (carotid to femoral); } \\
\text { peripheral PWV (femoral to } \\
\text { toe); left ventricular mass; end- } \\
\text { diastolic volume; end-systolic } \\
\text { volume; stroke volume; cardiac } \\
\text { output; ejection fraction; fractional } \\
\text { shortening. } \\
\text { Cardiovascular Indices-Head-up } \\
\text { Tilt: } \\
\text { Beat-by-beat BP, HR and R-R } \\
\text { interval; cvBRS. }\end{array}$ & $\begin{array}{l}\text { Psychosocial resources: } \\
\text { Perceived Stress Scale; self- } \\
\text { esteem; perceived mastery; } \\
\text { Optimism; minimisation; } \\
\text { Perfectionism; } \\
\text { Family social support. } \\
\text { Executive function } \\
\text { Mental health: } \\
\text { Psychological distress scale } \\
\text { (CESD); depression; anxiety; } \\
\text { hostility; aggression. } \\
\text { Personal strengths: strengths } \\
\text { knowledge; satisfaction with life. } \\
\end{array}$ & $\begin{array}{l}\text { Inflammatory biomarkers: } \dagger \\
\text { APRIL/TNFSF13; BAFF/ } \\
\text { TNFSF13B; sCD30/TNFRSF8; } \\
\text { sCD163; Chitinase-3-like 1; } \\
\text { gp130/sIL-6R } \beta \text {; IFN- } \alpha 2 ; \text { IFN- } \beta \text {; } \\
\text { IFN- } \gamma \text { I IL-2; sIL-6R } \alpha \text {; IL-8; IL-10; } \\
\text { IL-11; IL-12 (p40); IL-12 (p70); } \\
\text { IL-19; IL-20; IL-22; IL-26; IL- } \\
27 \text { (p28); IL-28A/IFN- } 22 ; \text { IL-29/ } \\
\text { IFN- } \lambda 1 ; \text { IL-32; IL-34; IL-35; LIGHT/ } \\
\text { TNFSF14; MMP-1; MMP-2; MMP- } \\
\text { 3; Osteocalcin; Osteopontin; } \\
\text { Pentraxin-3; sTNF-R1; sTNF-R2; } \\
\text { TSLP; TWEAK/TNFSF12. } \\
\text { C reactive protein } \\
\text { Chronic cortisol level: } \\
\text { Past 3-month scalp hair cortisol } \\
\text { level. } \\
\text { Cellular ageing biomarkers: } \\
\text { Telomere length; mitochondrial } \\
\text { DNA. } \\
\text { Brain derived neurotrophic factor. }\end{array}$ & $\begin{array}{l}\text { Physical activity. } \\
\text { Sleep behaviour: } \\
\text { Sleep problems; weekday and } \\
\text { weekend sleep duration; sleep } \\
\text { quality. } \\
\text { Food behaviour: } \\
\text { Food type consumption patterns; } \\
\text { eating habits. } \\
\text { Substance use: } \\
\text { Type of smoker; smoking } \\
\text { locations; secondhand smoke } \\
\text { exposure; other tobacco use; } \\
\text { current alcohol use; alcohol } \\
\text { frequency; current other illicit drug } \\
\text { use. } \\
\text { Personal medical history: } \\
\text { Medical diagnoses; current } \\
\text { prescription medication use; } \\
\text { current over-the-counter } \\
\text { medication use. } \\
\text { Family health history: } \\
\text { Hypertension; high cholesterol; } \\
\text { heart disease; stroke; type I } \\
\text { diabetes, type II diabetes. }\end{array}$ \\
\hline
\end{tabular}

${ }^{*}$ All acronyms in table are defined in the list of abbreviations.

†See http://www.bio-rad.com/en-ca/sku/171al001m-bio-plex-pro-human-inflammation-panel-1-37-plex?ID=171al001m

control group $(\mathrm{n}=63)$. This resulted in three annual waves of cardiovascular assessments, family demographics and family history of CVD and related diseases, anthropometric measures, physical activity, fasting lipid profiles and psychosocial and mental health measures up to grade 10 (16 years).

Study 3: Brock Active Muscles Study (BAM) was a longitudinal community study to examine physical activity in 291 participants from 8 to 18 years. The laboratory subsample conducted in 2009 was a 1-wave study of a random sample of the original 291 who were $8-18$ years and supplemented with other community participants (total $n=104$ ) to assess the effect of maturation on cardiovascular development. ${ }^{53}$ The battery of measures included non-invasive cardiovascular assessments, fasting lipid profiles, blood serum and some sociodemographic characteristics.

\section{Study protocol}

\section{Contacting participants and recruitment}

Available contact information is used from the three studies. However, this information is about 10 years old creating potential problems for follow-up for several reasons including families moving residences and changing phone numbers, families moving to cell phones and cancelling old 'land lines', and participants, now adults, no longer living at home. To address these issues and improve our recruitment rate, we are also employing a social media contact strategy. However, similar to using existing contact information, the use of social media recruitment may also lend itself to selective attrition as various groups of participants may not be captured. For example, while social media recruitment has greatly increased our ability to contact and retest participants who are otherwise inaccessible, we may still miss individuals who may have moved, become institutionalised, have developed serious health problems, are deceased or are generally unreachable. Selective attrition will be identified using baseline data and dealt with as required in analyses.

\section{Recruitment and laboratory visit}

On successful contact through phone or social media, we explain the study and testing protocol to participants. We inform them that they will receive a CAD100 honourarium to compensate them for their participation and travel to the university. In addition, for those coming from neighbouring cities outside the local area, we provide additional funding to compensate for added travel costs. Participants who have moved to other provinces or countries are offered a chance to complete testing while back visiting the region.

On scheduling the laboratory visit, participants are sent an information package (via mail, email or social media) containing a letter of introduction, directions to the university, a parking pass, confirmation of the scheduled testing time and laboratory contact information in case they need to cancel or reschedule. Participants are instructed to avoid vigorous physical activity, alcohol and caffeine for 12 hours and to fast for 4 hours prior to their visit. 
If a participant is or was recently sick or identifies any recent antibiotic use, the appointment is rescheduled to prevent bias due to potentially elevated or suppressed inflammation. Participants are also asked to contact the laboratory if they feel sick within 2 days after testing. On arrival for testing, the study is again described to them and informed consent is completed. Finally, we gain signed permission to agree to allow us to keep their data on file for future analyses and future contact to address additional research questions that may arise.

\section{Cardiovascular and anthropometric assessments}

Prior to data collection, participants are asked to void their bladder to prevent the effect of bladder distension on $\mathrm{BP}^{54}$ Afterwards, following 5 min of seated rest, six $\mathrm{BP}$ and $\mathrm{HR}$ measurements at $1 \mathrm{~min}$ intervals are taken using an automated oscillometric device (BpTRU Vital Signs Monitor, BPM-300, VSM, MedTech Devices , Coquitlam, BC, Canada). The final three measures are used to measure average $\mathrm{BP}$ and $\mathrm{HR}^{55-57}$ Standing height is measured $(\mathrm{cm})$ using a stadiometer (STAT-7X, Ellard Instrumentations, Monroe, Washington). Body mass $(\mathrm{kg})$ is measured with a digital scale (BWB-800S, Tanita, Tokyo, Japan). Waist and hip circumferences $(\mathrm{cm})$ are measured at the iliac crest and widest gluteal protuberance, respectively. Fat mass, fat-free mass and body fat percentage is measured via air-displacement plethysmography (BODPOD, Life Measurement, Concord, California, USA) 5859

Participants then undergo a series of non-invasive cardiovascular measures. Participants lay at rest in a supine position in a quiet, dimly lit room for all cardiovascular testing. Participants are outfitted with a standard single lead ECG for continuous R-R interval (ie, HR) measurement throughout the data collection period. Participants are outfitted with a finger BP cuff (Nexfin, BMEYE, Amsterdam, Horton, Norway; NIBP Nano, ADInstruments, Colorado Springs, USA) placed on the left middle finger to assess beat-by-beat BP throughout the duration of testing. Supine SBP and DBP are measured manually using a sphygmomanometer and stethoscope at the right brachial artery. Afterwards, $10 \mathrm{~min}$ of beat-by-beat R-R interval and BP data are collected. Beat-by-beat finger BPs are adjusted to the average manually obtained supine SBP and DBP as BP obtained at the finger overestimates brachial BP. Cardiovagal baroreflex sensitivity (cvBRS) is measured using beat-by-beat R-R interval (time between successive R-waves) and SBP. Matlab software is used to run a Fast Fourier Transform to determine low frequency (LF) (LF: 0.04-0.15 Hz) and high frequency (HF) (HF: $0.15-0.4 \mathrm{~Hz}$ ). We use a transfer function calculation of the LF region of SBP to R-R interval to measure cvBRS. ${ }^{52} 53$

Measures of arterial stiffness and thickness include pulse wave velocity (PWV), common carotid artery (CCA) distensibility and compliance, and IMT. Using a hand-held tonometer to obtain pressure waveforms (Millar Instruments, Houston, USA), PWV is determined for both central (carotid-to-femoral) and peripheral (femoral-to-toe) vascular segments. ${ }^{60}$ Distensibility is measured using the CCA due to its anatomical accessibility, imaging capabilities and anatomical link to the carotid baroreceptors. ${ }^{61}$ The right CCA is visually assessed with B-mode ultrasound video clips and edge-tracking software. Edge tracking software (MAUI, Hedgehog Medical, Waterloo, Canada) is used to determine changes in arterial diameter during systole and diastole of the CCA. Change in arterial diameter and CCA pulse pressure are used to calculate compliance and distensibility. CCA IMT, a measure of arterial wall thickness, is measured on the far (deep) wall as it consistently provides more clarity. Cardiac size and function are measured and analysed using non-invasive echocardiography following American Society of Echocardiography guidelines. Images are taken using the parasternal long-axis view, which optimises the left ventricle, while participants lay in a supine position. Calculations of LVM are done using a validated equation indexed to height 2.7, which identifies left ventricular hypertrophy. ${ }^{62}$ We estimate stroke volume, cardiac output, ejection fraction and fractional shortening. Finally, participants are subjected to a $60^{\circ}$ head-up-tilt (HUT) to assess the ability of the body to respond an orthostatic-induced stressor. Throughout the HUT procedure, beat-by-beat $\mathrm{R}-\mathrm{R}$ interval and BP are collected to examine cardiovascular adaptations to HUT. ${ }^{63}$

\section{Stress biomarkers}

Blood, hair and saliva samples are then collected by a registered nurse. Blood is collected from the antecubital fossa of participants into two $5 \mathrm{~mL}$ SSTTM Serum Separation Tubes (BD Biosciences, \#367986, Franklin Lakes, New Jersey, USA). Blood is given $30 \mathrm{~min}$ to clot at room temperature before being centrifuged at $3000 \mathrm{x}$ g for $15 \mathrm{~min}$ at $4^{\circ} \mathrm{C}$. Following centrifugation, serum is aliquoted and stored at $-80^{\circ} \mathrm{C}$ until analysis. Inflammatory markers, including cytokines and chemokines, are assessed using a custom magnetic bead-based multiplexing antibody assay (Bio-Rad) and analysed on a MagPix (Luminex) ${ }^{64}$ instrument. We measure 37 inflammatory markers on a multiplex panel across four domains including TNF superfamily proteins, IFN family proteins, and T-cell cytokines including $\mathrm{T}$ regulatory and matrix metalloproteinases. CRP levels are analysed using a Human CRP Quantikine ELISA kit (R\&D Systems, \#DCRP00, Minneapolis, Minnesota, USA). Chronic glucocorticoid cortisol level is measured by processing hair samples to extract cortisol and employing a biochemical colorimetric assay. ${ }^{6566}$ Assuming a $1 \mathrm{~cm}$ per month growth rate, we harvest a pencil-width sample of the first $3 \mathrm{~cm}$ of hair closest to the scalp providing a measure of 3-month chronic cortisol levels. Telomere length is assessed from saliva samples through DNA extraction using quantitative PCR. ${ }^{49}$

\section{Self-report questionnaire}

Due to the total length of the visit, after laboratory testing, participants are given a short break and are given a snack and beverage. They then complete a standardised questionnaire that is self-reported to minimise discomfort 
and social response bias due to the sensitivity of questions. The questionnaire takes about 1 hour to complete at their own pace. Those recruited through social media are also given a research ethics board mandated social media questionnaire supplement. To maintain confidentiality, the questionnaire is given to the participant in an envelope with only their unique study ID. Participants are informed a third party with no access to personal identifiers will enter all data. The research coordinator is unobtrusively present in the room to assist with any clarification required by the participant.

Questions focus on both positive and negative lifestyle, behavioural, psychosocial and mental health measures identified in the CVH, ACEs and stress process literature..$^{35} 3767$ Many measures were already collected at baseline to allow for analyses of change (tables 1 and 2). Measures include physical activity and sedentary behaviour (International Physical Activity Questionnaire), ${ }^{68}$ sleep problems and sleep duration, ${ }^{69}{ }^{70}$ food and eating habits, depression (Centre for Epidemiological Studies-Depression (CES-D)) ${ }^{71}$ anxiety and hostility (Symptom Checklist-90-Revised (SCL-90-R)), ${ }^{72}$ aggression, ${ }^{73}$ stress appraisal (Perceive Stress Scale (PSS) $),{ }^{74}$ psychosocial resources including self-esteem, ${ }^{75}$ perceived mastery, ${ }^{76}$ family social support and attachment, and executive function (Behaviour Rating Inventory of Executive Function (BRIEF)), ${ }^{77}$ and self-reported medical diagnoses and medications, and family history of CVD, high BP and diabetes. Additional measures include smoking, alcohol and illicit drug use, satisfaction with life, ${ }^{78}$ knowledge of personal strengths ${ }^{79}$ and perfectionism. ${ }^{80}$ We have baseline data on sociodemographic information reported by parents to control for heterogeneity across studies. We collect current sociodemographic information from study participants, specifically marital status, participant and spouse (if applicable) ethnicity, current occupation and/or schooling, education level and finally current living arrangements and a number of children living both with the participant or away from the participant. In addition to the primary focus on the ACEs-CVH relationship, our questionnaire components also allow for investigation of cumulative, mediating and/or moderating influences of other, previously identified childhood risk factors. ${ }^{29-34}$

To ensure that ACE questions do not influence responses or prompt non-responses to subsequent questions, this component appears near the end of the questionnaire. We use two ACEs instruments to ensure complete capture. First, the Childhood Trust Events Survey-2.0 is a measure of ACEs for children and young adults modelled after the original Kaiser ACEs inventory ${ }^{2}$ providing information on abuse and household dysfunctions ${ }^{81}$ but adding additional childhood stressful experiences including bullying, violent neighbourhood crime, separation from parents and family, severe material disadvantage, homelessness, natural disasters and serious illnesses, accidents or injuries. Second, the Child Trauma Questionnaire, ${ }^{82}$ validated for young adult Canadian undergraduates directly relevant to our study population, asks multiple questions for each of the five dimensions of abuse to facilitate recall in screening for trauma. ${ }^{82}$ However, it does not capture anything about household dysfunction or other exposures. A potential concern in ACEs research is the willingness of participants to report experiences. However, researchers report that when conducted in a dignified manner, there is a high level of compliance, reliability and accuracy in reporting child maltreatment, household dysfunction, and other ACEs ${ }^{21} 81$ with kappas of $0.6-0.7$ over 1 year. ${ }^{81}$ In contrast, a recent meta-analysis identifies poor overall agreement between prospective and retrospective assessments of childhood adversity, ${ }^{83} \mathrm{a}$ limitation endemic to much of the current research on ACEs. We attempt to minimise this issue by including two instruments to increase the capture rate of ACEs and ask participants while still young adults lessening the length of time for retrospective recall in comparison to that of many published studies in the field.

\section{Concluding the laboratory visit}

After completing the questionnaire, the participant places it in the envelope and seals it to ensure confidentiality. Participants are given an opportunity to express concerns about any of the questions verbally acknowledging that some may be distressing. The research coordinator also asks if the participant feels a need to speak to someone about any issues raised regarding the questions and informs them that we have established referral resources through Family Counselling Centre Niagara (FCC). An FCC pamphlet is attached to the back of the questionnaire for participants to take home outlining the confidential counselling available for issues such as past trauma, depression and anxiety. The FCC provides a free walk-in clinic for immediate and emergency services funded by the United Way. It is the participant's decision to use FCC or any other counselling services.

At the end of the lab visit, participants are given their CAD100 honorarium and any additional reimbursement expenses for travel and thanked for participating.

\section{Patient and public involvement}

There was no patient or public involvement in this study protocol.

\section{DISCUSSION}

The motivation for this study is based on our preliminary work examining parent-reported ACEs using the baseline HBEAT study. While unable to examine the full spectrum of ACEs using HBEAT, specifically child abuse and maltreatment, this work linked a toxic childhood environment to obesity, larger waist circumference and elevated HR, but interestingly, not elevated SBP. ${ }^{51}$ As well, using the cardiovascular assessment data, we found a connection between ACEs and systemic arterial stiffness but only among boys. ${ }^{52}$ Based on these findings and other work examining ACEs and CVH and CVD, the NLHS builds on this work to prospectively test the effect of ACEs on cardiovascular 
development from childhood to early adulthood. Integrating the stress process and biological embedding theoretical frameworks, the NLHS study design will permit the investigation of the cumulative, mediating and/or moderating effects between ACEs and other childhood risk factors with stress biomarkers on CVH. To our knowledge, the effect of ACEs on preclinical CVH indices has not been studied in a prospective manner from childhood to early adulthood nor has there been efforts to identify the psychosocial and physiological mediating and moderating pathways. The importance of incorporating this multidimensional focus is clearly supported by recent ACEs research and reviews showing that previously identified traditional and behavioural risk factors including family history of disease, smoking, physical activity, alcohol use and obesity, while important in their own right, do not explain away the relationship between ACEs and CVD. ${ }^{4-11}$ This study places us in a novel position given the current state of literature, the age of our cohorts, and the potentially large sample to examine changes in $\mathrm{CVH}$ from childhood to early adulthood associated with ACEs.

\section{Author affiliations}

${ }^{1}$ Health Sciences, Brock University, St. Catharines, Ontario, Canada

${ }^{2}$ Brock-Nlagara Centre for Health and Well-Being, Brock University, St. Catharines, Ontario, Canada

${ }^{3}$ Child and Youth Studies, Brock University, St. Catharines, Ontario, Canada ${ }^{4}$ Department of Psychology, Concordia University, Montreal, Quebec, Canada ${ }^{5}$ Faculty of Kinesiology and Physical Education, University of Toronto, Toronto, Ontario, Canada

Acknowledgements The authors acknowledge Jessy Moore, the current project coordinator for the NLHS as well as graduate students Aindriu McGuire, Kingston Wong, Talha Rafiq, Megan Henry and Nathan lannarelli for their work on this project.

Contributors TJW, DDO and JC are the principal investigators for the funded project and were responsible for the baseline studies guiding this work, the conceptualisation and design of the work and the interpretation of the data. KSD was the project coordinator for the pilot study, is responsible for collecting all cardiovascular measures, their analysis and interpretation and has contributed to the conceptualisation and design of the orthostatic stress component of the study. AJM, DSM and JJM all made substantial contributions to the conceptual and design aspects of both the psychosocial and physiological components of the work and all will be involved in the analysis and interpretation of aspects pertinent to their expertise. All authors were involved in writing and editing the CIHR-funded NLHS research proposal. TJW took the lead in revising the grant proposal for publication. All authors contributed in editing and reviewing the manuscript, approved of the final submission and agreed to be personally accountable for their specific contributions.

Funding The NLHS is supported by the Canadian Institutes of Health Research (CIHR) (award \#'s 363774, 399332). HBEAT was supported by the Ontario Heart and Stroke Foundation (HSFO award \#SDA6237). PHAST was supported by CIHR (award \#66959). BAM was supported by CIHR (award \# 199944).

Competing interests None declared.

Patient consent for publication Not required.

Ethics approval Ethics approval has been received from Brock University BREB (Biological Research Ethics Board), file numbers 16-078-WADE and 18-288- WADE. To improve our recruitment rate, we received ethics approval to employ a social media strategy adhering to specific parameters mandated by the University Research Ethics Board (REB) to respect data privacy and confidentiality.

Provenance and peer review Not commissioned; externally peer reviewed.
Open access This is an open access article distributed in accordance with the Creative Commons Attribution Non Commercial (CC BY-NC 4.0) license, which permits others to distribute, remix, adapt, build upon this work non-commercially, and license their derivative works on different terms, provided the original work is properly cited, appropriate credit is given, any changes made indicated, and the use is non-commercial. See: http://creativecommons.org/licenses/by-nc/4.0/.

\section{REFERENCES}

1. Felitti VJ. Adverse childhood experiences and adult health. Acad Pediatr 2009;9:131-2.

2. Felitti VJ, Anda RF, Nordenberg D, et al. Relationship of childhood abuse and household dysfunction to many of the leading causes of death in adults. The Adverse Childhood Experiences (ACE) Study. Am J Prev Med 1998;14:245-58.

3. Barr RG, Fleming PA, Macmillan $\mathrm{H}$, et al. The Royal Society of Canada \& The Canadian Academy of Health Sciences Expert Panel: Early Childhood Development, 2012.

4. Basu A, McLaughlin KA, Misra S, et al. Childhood maltreatment and health impact: the examples of cardiovascular disease and type 2 diabetes mellitus in adults. Clin Psychol 2017;24:125-39.

5. Hughes K, Bellis MA, Hardcastle KA, et al. The effect of multiple adverse childhood experiences on health: a systematic review and meta-analysis. Lancet Public Health 2017;2:e356-66.

6. Fuller-Thomson E, Brennenstuhl S, Frank J. The association between childhood physical abuse and heart disease in adulthood: findings from a representative community sample. Child Abuse Negl 2010;34:689-98.

7. Dong M, Giles WH, Felitti VJ, et al. Insights into causal pathways for ischemic heart disease: adverse childhood experiences study. Circulation 2004;110:1761-6.

8. Kiecolt-Glaser JK, Gouin JP, Weng NP, et al. Childhood adversity heightens the impact of later-life caregiving stress on telomere length and inflammation. Psychosom Med 2011;73:16-22.

9. Slopen N, Kubzansky LD, McLaughlin KA, et al. Childhood adversity and inflammatory processes in youth: a prospective study. Psychoneuroendocrinology 2013;38:188-200.

10. D'Andrea W, Sharma R, Zelechoski AD, et al. Physical health problems after single trauma exposure: when stress takes root in the body. J Am Psychiatr Nurses Assoc 2011;17:378-92.

11. Steptoe A, Hamer M, Chida Y. The effects of acute psychological stress on circulating inflammatory factors in humans: a review and meta-analysis. Brain Behav Immun 2007;21:901-12.

12. Afifi TO, MacMillan HL, Boyle M, et al. Child abuse and mental disorders in Canada. Can Med Assoc J 2014;186:E324-32.

13. MacDonald STS. Alberta adverse childhood experiences survey. Calgary, Alberta: ACCFCR, 2014.

14. Tonmyr L, Ouimet $C$, Ugnat A. A review of findings from the canadian incidence study of reported child abuse and Neglect (CIS). Can J Public Heal 2012;103:103-12.

15. Surtees PG, Wainwright NW, Luben RL, et al. Adaptation to social adversity is associated with stroke incidence: evidence from the EPIC-Norfolk prospective cohort study. Stroke 2007;38:1447-53.

16. Su S, Wang X, Pollock JS, et al. Adverse childhood experiences and blood pressure trajectories from childhood to young adulthood: the Georgia stress and Heart study. Circulation 2015;131:1674-81.

17. Thomas C, Hyppönen E, Power C. Obesity and type 2 diabetes risk in midadult life: the role of childhood adversity. Pediatrics 2008;121:e1240-9.

18. Surtees PG, Wainwright NW. The shackles of misfortune: social adversity assessment and representation in a chronic-disease epidemiological setting. Soc Sci Med 2007;64:95-111.

19. Widom CS, Raphael KG, Dumont KA. The case for prospective longitudinal studies in child maltreatment research: commentary on Dube. Child Abuse Negl 2004;28:715-22.

20. Colman I, Kingsbury M, Garad Y, et al. Consistency in adult reporting of adverse childhood experiences. Psychol Med 2016;46:543-9.

21. Dube SR, Williamson DF, Thompson T, et al. Assessing the reliability of retrospective reports of adverse childhood experiences among adult $\mathrm{HMO}$ members attending a primary care clinic. Child Abuse Negl 2004;28:729-37.

22. Li S, Chen W, Srinivasan SR, et al. Childhood cardiovascular risk factors and carotid vascular changes in adulthood. JAMA 2003;290:2271-943.

23. Rocchini AP, Freedman DS. Childhood obesity and coronary heart disease. N Engl J Med 2011;365:160-9.

24. Raitakari OT, Juonala M, Kahonen M, et al. Cardiovascular risk factors in childhood and carotid artery intima-media thickness 
in adulthood: the cardiovascular risk in young finns study. JAMA 2015;290:2277-83.

25. Ben-Shlomo Y, Spears M, Boustred C, et al. Aortic pulse wave velocity improves cardiovascular event prediction: an individual participant meta-analysis of prospective observational data from 17,635 subjects. J Am Coll Cardiol 2014;63:636-46.

26. Bouzas-Mosquera A, Broullón FJ, Álvarez-García N, et al. Association of left ventricular mass with all-cause mortality, myocardial infarction and stroke. PLoS One 2012;7:e45570-6.

27. Kiviniemi AM, Tulppo MP, Hautala AJ, et al. Prognostic significance of impaired baroreflex sensitivity assessed from Phase IV of the Valsalva maneuver in a population-based sample of middle-aged subjects. Am J Cardiol 2014;114:571-6.

28. Miller GE, Chen E, Parker KJ. Psychological stress in childhood and susceptibility to the chronic diseases of aging: moving toward a model of behavioral and biological mechanisms. Psychol Bull 2011;137:959-97.

29. Elovainio M, Hakulinen C, Pulkki-Råback L, et al. A network approach to the analysis of psychosocial risk factors and their association with health. J Health Psychol 2018:135910531876562-14.

30. Evans GW, Li D, Whipple SS. Cumulative risk and child development. Psychol Bull 2013;139:1342-96.

31. Appleton AA, Holdsworth E, Ryan M, et al. Measuring childhood adversity in life course cardiovascular research: a systematic review. Psychosom Med 2017;79:434-40.

32. Hakulinen C, Pulkki-Råback L, Elovainio M, et al. Childhood psychosocial cumulative risks and carotid intima-media thickness in adulthood: the cardiovascular risk in young finns study. Psychosom Med 2016;78:171-81.

33. Non AL, Rewak M, Kawachi I, et al. Childhood social disadvantage, cardiometabolic risk, and chronic disease in adulthood. Am J Epidemiol 2014;180:263-71.

34. Juonala M, Pulkki-Råback L, Elovainio $M$, et al. Childhood psychosocial factors and coronary artery calcification in adulthood: the cardiovascular risk in young finns study. JAMA Pediatr 2016;170:466-72.

35. Pearlin LI, Lieberman MA, Menaghan EG, et al. The stress process. $J$ Health Soc Behav 1981;22:337-56.

36. Foster $\mathrm{H}$, Brooks-Gunn J. Toward a stress process model of children's exposure to physical family and community violence. Clin Child Fam Psychol Rev 2009;12:71-94.

37. Pearlin LI. The stress process revisited: reflections on concepts and their interrelationships, 1999:395-415.

38. De Bellis MD. Developmental traumatology: the psychobiological development of maltreated children and its implications for research, treatment, and policy. Dev Psychopathol 2001;13:539-64.

39. De Bellis MD, Zisk A. The biological effects of childhood trauma. Child Adolesc Psychiatr Clin N Am 2014;23:185-222.

40. Zhang TY, Labonté B, Wen XL, et al. Epigenetic mechanisms for the early environmental regulation of hippocampal glucocorticoid receptor gene expression in rodents and humans. Neuropsychopharmacology 2013;38:111-23.

41. McGowan PO, Sasaki A, D'Alessio AC, et al. Epigenetic regulation of the glucocorticoid receptor in human brain associates with childhood abuse. Nat Neurosci 2009;12:342-8.

42. Coe CL, Lubach GR. Prenatal origins of individual variation in behavior and immunity. Neurosci Biobehav Rev 2005;29:39-49.

43. Coe CL, Lubach GR. Mother-infant interactions and the development of immunity from conception through weaning. Vol. I, Psychoneuroimmunology. 4th edn: Elsevier Inc, 2007:455-74.

44. Danese A, Pariante CM, Caspi A, et al. Childhood maltreatment predicts adult inflammation in a life-course study. Proceedings of the National Academy of Sciences 2007;104:1319-24.

45. Carpenter LL, Gawuga CE, Tyrka AR, et al. Association between plasma IL-6 response to acute stress and early-life adversity in healthy adults. Neuropsychopharmacology 2010;35:2617-23.

46. Foege WH. Adverse childhood experiences. A public health perspective. Am J Prev Med 1998;14:354-5.

47. Lee C, Tsenkova V, Carr D. Childhood trauma and metabolic syndrome in men and women. Soc Sci Med 2014;105:122-30.

48. Shalev I, Moffitt TE, Sugden K, et al. Exposure to violence during childhood is associated with telomere erosion from 5 to 10 years of age: a longitudinal study. Mol Psychiatry 2013;18:576-81.

49. Puterman E, Gemmill A, Karasek D, et al. Lifespan adversity and later adulthood telomere length in the nationally representative US Health and Retirement Study. Proc Natl Acad Sci U S A 2016;113:E6335-42.

50. Yeh J-K, Wang C-Y. Telomeres and telomerase in cardiovascular diseases. Genes 2016;7:58-18.

51. Pretty C, O'Leary DD, Cairney J, et al. Adverse childhood experiences and the cardiovascular health of children: a crosssectional study. BMC Pediatr 2013;13:208.
52. Klassen SA, Chirico D, O'Leary DD, et al. Linking systemic arterial stiffness among adolescents to adverse childhood experiences. Child Abuse Negl 2016;56:1-10.

53. Chirico D, Liu J, Klentrou P, et al. The effects of sex and pubertal maturation on cardiovagal baroreflex sensitivity. J Pediatr 2015;167:1067-73.

54. Fagius J, Karhuvaara S. Sympathetic activity and blood pressure increases with bladder distension in humans. Hypertension 1989;14:511-7.

55. Coverdale NS, Fitzgibbon LK, Reid GJ, et al. Baroreflex sensitivity is associated with sleep-related breathing problems in adolescents. $J$ Pediatr 2012;160:610-4.

56. Fitzgibbon LK, Coverdale NS, Phillips AA, et al. The association between baroreflex sensitivity and blood pressure in children. Appl Physiol Nutr Metab 2012;37:301-7.

57. Phillips AA, Chirico D, Coverdale NS, et al. The association between arterial properties and blood pressure in children. Appl Physiol Nutr Metab 2015;40:72-8.

58. Fields DA, Goran MI, McCrory MA. Body-composition assessment via air-displacement plethysmography in adults and children: a review. Am J Clin Nutr 2002;75:453-67.

59. Noreen EE, Lemon PW. Reliability of air displacement plethysmography in a large, heterogeneous sample. Med Sci Sports Exerc 2006;38:1505-9.

60. Mackenzie IS, Wilkinson IB, Cockcroft JR. Assessment of arterial stiffness in clinical practice. QJM 2002;95:67-74

61. O'Leary DD, Steinback CD, Cechetto AD, et al. Relating druginduced changes in carotid artery mechanics to cardiovagal and sympathetic baroreflex control. Can J Physiol Pharmacol 2005;83:439-46.

62. de Simone G, Devereux RB, Daniels SR, et al. Stroke volume and cardiac output in normotensive children and adults. Circulation 1997:95:1837-43.

63. Smith JJ, Porth CM, Erickson M. Hemodynamic response to the upright posture. J Clin Pharmacol 1994;34:375-86.

64. R\&D Systems. Luminex® assays and luminex high performance assays. https://www.rndsystems.com/products/luminex-assays-andhigh-performance-assays

65. Stalder T, Kirschbaum C. Analysis of cortisol in hair--state of the art and future directions. Brain Behav Immun 2012;26:1019-29.

66. Russell E, Koren G, Rieder M, et al. Hair cortisol as a biological marker of chronic stress: current status, future directions and unanswered questions. Psychoneuroendocrinology 2012;37:589-601.

67. Sood E, Gidding SS. Childhood psychosocial determinants of cardiovascular health. Curr Cardiovasc Risk Rep 2016;10:1-8

68. Craig CL, Marshall AL, Sjöström M, et al. International physical activity questionnaire: 12 -country reliability and validity. Med Sci Sports Exerc 2003;35:1381-95

69. Spoormaker VI, Verbeek I, van den Bout J, et al. Initial validation of the SLEEP-50 questionnaire. Behav Sleep Med 2005;3:227-46.

70. Morin CM, Savard J, Ouellet MC. Insomnia: John Wiley Sons, Inc, 1993.

71. Radloff LS. The CES-D scale: a self-report depression scale for research in the general population. Appl Psychol Meas 1977;1:385-401.

72. Derogatis LR, Unger R. Symptom checklist-90-revised. Corsini encyclopedia of psychology, 2010.

73. Achenbach TM, Dumenci L, Rescorla LA, et al. DSM-oriented and empirically based approaches to constructing scales from the same item pools DSM -oriented and empirically based approaches to constructing scales from the same item pools. J Clin Child Adolesc Psychol 2010;32:37-41.

74. Jacobs PD, Munz DC. An index for measuring perceived stress in a college population. J Psychol 1968;70:9-15

75. Rosenberg M. Conceiving of self. New York: Basic Books, 1979.

76. Pearlin LI, Schooler C. The structure of coping. J Health Soc Behav 1978;19:2-21.

77. Roth RM, Isquith PK, Gioia G. BRIEF-A: behavior rating inventory of executive function - adult version, professional manual. Psychol Assess Resour 2005

78. Diener E, Emmons RA, Larsen RJ, et al. The satisfaction with life scale. J Pers Assess 1985;49:71-5.

79. Govindji R, Linley PA. Strengths use, self-concordance and well-being, Implications for strengths coaching and coaching psychologists. Int Coach Psychol Rev 2007;2:143-53.

80. Flett GL, Hewitt PL, Blankstein KR, et al. Psychological distress and the frequency of perfectionistic thinking. J Pers Soc Psychol 1998;75:1363-81.

81. Edwards VJ, Dube SR, Felitti VJ, et al. It's ok to ask about past abuse. Am Psychol 2007;62:327-8. 
82. Bernstein DP, Ahluvalia T, Pogge D, et al. Validity of the childhood trauma questionnaire in an adolescent psychiatric population. $J \mathrm{Am}$ Acad Child Adolesc Psychiatry 1997;36:340-8.
83. Baldwin JR, Reuben A, Newbury JB, et al. Agreement between prospective and retrospective measures of childhood maltreatment: a systematic review and meta-analysis. JAMA Psychiatry 2019:1-10. 